\title{
Meduloblastomas del adulto: serie quirúrgica de 11 casos
}

\author{
R. Rodríguez-Mena; R.A. Barbella-Aponte; J.M. Gallego-Sánchez y C. Barcia-Mariño
}

Servicio de Neurocirugía. Servicio de Anatomía Patológica. Consorcio Hospital General Universitario de Valencia. Valencia. España.

Resumen

Introducción. Los meduloblastomas son tumores malignos, invasivos, altamente celulares, del cerebelo, infrecuentes en el adulto. En el presente trabajo describiremos la experiencia en el manejo de esta patología en nuestro centro.

Material y métodos. Se trata de un trabajo retrospectivo que incluyó 11 pacientes adultos con diagnóstico de meduloblastoma del eje craneoespinal atendidos entre 1984-2010. Se evaluaron datos clínicos, radiológicos y terapéuticos durante la evolución de la enfermedad.

Resultados. La edad media de los pacientes fue 30,2 años (9 mujeres y 2 varones). Más del $80 \%$ de los pacientes manifestaron clínica de hipertensión intracraneal, mientras el $54,5 \%$ presentaron síndrome cerebeloso. El grado de resección quirúrgica fue total en 8 pacientes $(72,7 \%)$ y subtotal en $3(27,3 \%)$. Todos los pacientes recibieron tratamiento radioterápico adyuvante. Posterior a la exéresis tumoral inicial, 6 pacientes recibieron quimioterapia adyuvante con cisplatino (CDDP) y etopósido (VP-16). Durante el seguimiento, después de un tiempo medio de 35,2 meses, se observaron recidivas en el $36,4 \%$ de los pacientes $(n=4)$, con mayor frecuencia en la fosa posterior, empleando en todos ellos tratamiento con cirugía y quimioterapia. El tiempo medio de supervivencia fue 100,3 meses, con un índice de supervivencia estimado a los 5 y 8 años de seguimiento del 84 y $56 \%$ respectivamente.

Conclusiones. La máxima resección quirúrgica forma parte crucial del tratamiento, seguida de adyuvancia oncológica tan pronto sea posible, con radioterapia seguida o no de quimioterapia.

PALABRAS CLAVE. Adulto. Cirugía. Meduloblastoma. Quimioterapia. Radioterapia craneoespinal.

Adult medulloblastomas: our experience

Recibido: 23-05-11. Aceptado: 7-07-11.
Summary

Introduction. Medulloblastomas are malignant, invasive and highly cellular tumours from the cerebellum, rarely seen in adults. We describe the experience in the treatment of this entity in our institution.

Materials and methods. A retrospective study was made including 11 adult patients with medulloblastomas confined to the craniospinal axis treated between 1984-2010. Clinical, radiological and therapeutic data were assessed during the evolution of this entity.

Results. Mean age of patients was 30,2 years ( 9 women $/ 2$ men). Over $80 \%$ of the patients presented intracranial hypertension, while $54,5 \%$ presented cerebellum syndrome. Gross total surgical resection was achieved in 8 patients $(72,7 \%)$ and subtotal resection in the other $3(27,3 \%)$. All patients received craniospinal radiotherapy. After primary surgical resection, 6 patients received chemotherapy with cisplatin (CDDP) and etoposide (VP-16). During the follow up period and after a median time of 35,2 months, 4 patients $(36,4 \%)$ presented with relapse, mainly in the posterior cranial fossa, managed in the majority of cases with surgical resection plus additional chemotherapy. Mean survival time was 100,3 months with a 5- and 8- year overall survival rate of 84 and $56 \%$ respectively.

Conclusions. In the treatment of this malignancy, gross surgical resection has a crucial role, followed as soon as possible by oncological therapy, specially radiotherapy and chemotherapy if needed.

KEY WORDS. Adult. Chemotherapy. Craniospinal radiotherapy. Medulloblastoma. Surgery.

Abreviaturas. CDDP: cisplatino. DVP: derivación ventriculoperitoneal. ECOG: Eastern Cooperative Oncology Group. IRM: imagen de resonancia magnética. LCR: Líquido cefalorraquídeo. QT: quimioterapia. RT: radioterapia. TC: tomografía computarizada. VP-16: etopósido. 


\section{Introducción}

Los meduloblastomas son tumores malignos, invasivos, altamente celulares, de la fosa craneal posterior, que se encuentran formando parte del grupo de tumores embrionarios según la Organización Mundial de la Salud ${ }^{35}$, con tendencia a metastizar en el neuroeje siguiendo las vías del líquido cefalorraquídeo (LCR).

Constituyen entre el 15 y $25 \%$ de los tumores cerebrales primarios de la población pediátrica ${ }^{40}$. Sin embargo, en el adulto representan un $1 \%$ de los tumores cerebrales ${ }^{37}$, apareciendo en un $80 \%$ de los casos antes de los 40 años ${ }^{6}$. Por ello existen pocas series de pacientes adultos con esta enfermedad, en su mayoría series muy cortas.

Existen algunas diferencias en las características de estos tumores entre la población pediátrica y adulta; en ésta última es más frecuente la localización hemisférica, presente en aproximadamente el 50\% de los $\operatorname{casos}^{3,4,9,11}$, y la variante histológica desmoplásica ${ }^{8,11,23,33}$, así como también difieren en su evolución y pronóstico ${ }^{36}$.

La forma de presentación clínica predominante es a través de un síndrome de hipertensión intracraneal (especialmente si asocia obstrucción de la circulación del LCR), acompañado de focalidad cerebelosa.

Las recomendaciones actuales sobre el manejo terapéutico de pacientes adultos con meduloblastomas se basan esencialmente en estudios retrospectivos en niños, tomando en cuenta 3 pilares fundamentales: cirugía resectiva, radioterapia (RT) y quimioterapia (QT). Por otro lado, definir factores pronósticos en el caso de adultos con meduloblastomas es un tema controvertido, basado en la heterogeneidad de los datos encontrados en los principales estudios realizados 5 .

En el presente trabajo describiremos las características de los pacientes adultos con meduloblastomas atendidos en nuestro centro, la experiencia en el manejo terapéutico de esta entidad poco frecuente desde el diagnóstico y durante su evolución, con el objetivo de determinar los resultados clínicos a corto y mayor plazo.

\section{Material y métodos}

Se trata de un trabajo de tipo retrospectivo que incluyó la totalidad de pacientes con edad $\geq 18$ años con diagnóstico de meduloblastoma del eje craneoespinal, con confirmación anatomopatológica $(\mathrm{n}=11)$, quienes han sido tratados en el período comprendido entre Agosto de 1984 y Agosto de 2010 en el Consorcio Hospital General Universitario de Valencia. Para la identificación de los casos se llevó a cabo una búsqueda en la base de datos del servicio de anatomía patológica (existente desde el año 1984). Posteriormente se realizó una revisión de las historias clínicas de los pacientes, recogiendo información en relación a la edad, sexo, síntomas, localización de la lesión, grado de resección quirúrgica, complicaciones postoperatorias, clasificación anatomopatológica, tratamiento adyuvante oncológico y su toxicidad, evolución de la enfermedad y seguimiento.

El manejo de los pacientes fue realizado conjuntamente entre neurocirujanos, oncólogos y radioterapeutas.

Se realizó un estudio clínico y radiológico de los pacientes, inicialmente con tomografía (TC) cerebral y a continuación imágenes de resonancia magnética (IRM) cerebral, con excepción de un caso intervenido en 1984, en el cual el estudio de imagen se limitó a la TC cerebral. Los signos y síntomas de presentación de los pacientes se agruparon en 2 grupos: síndrome de hipertensión intracraneal (si estaban presentes 3 o más de los siguientes: cefalea, vómitos, visión borrosa, fotofobia, diplopia y disminución del nivel de consciencia) y síndrome cerebeloso (si estaban presentes dos o mas de los siguientes: ataxia, nistagmus, disartria y vértigo).

El estudio anatomopatológico incluyó además del análisis histológico, un protocolo de inmunohistoquimia aplicado a todos los casos desde 1990 (9 pacientes) que incluía tinciones con enolasa neuroespecífica, proteína gliofibrilar ácida (GFAP), sinaptofisina y medición del índice de proliferación celular con la tinción nuclear de Ki67. Posterior a la confirmación anatomopatológica del tumor, se completó el estudio mediante análisis de LCR, obtenido mediante punción lumbar, y pruebas de neuroimagen del resto del eje craneoespinal (principalmente IRM). De esta manera y para fines del presente estudio, se distribuyeron a los pacientes según la escala del estado funcional de la Eastern Cooperative Oncology Group (ECOG) ${ }^{11}$ y según el sistema de estadiaje tumoral de Chang 5 .

Todos los pacientes recibieron tratamiento quirúrgico, mediante abordaje de fosa posterior a través de craniectomía suboccipital en línea media o lateral según la localización del tumor. El grado de resección tumoral, total (si fuese $>90 \%$ ) o subtotal (entre el 50-90\%), se basó en la revisión de protocolos quirúrgicos, acompañado de IRM cerebral (en 6 pacientes) o TC Cerebral (en 5 pacientes) realizadas en las primeras 72 horas del postoperatorio.

A continuación de la cirugía resectiva, los pacientes recibieron tratamiento adyuvante con RT y/o QT, según acuerdo del comité de neurooncología de nuestro centro. En este sentido, en la mayoría de los casos, se consideraron pacientes preferentes para QT postoperatoria aquellos con estadio $\geq$ T3a de Chang (incluyendo diseminación a través del LCR), pacientes con resección subtotal de la lesión y/o episodios de recidiva tumoral. El protocolo de QT seguido en nuestro centro incluyó la administración del tratamiento tanto previo como posterior a la RT, en función de la respuesta y tolerancia a la radiación.

El tratamiento radioterápico se aplicó sobre el eje craneoespinal con una sobreimpresión en fosa posterior. 


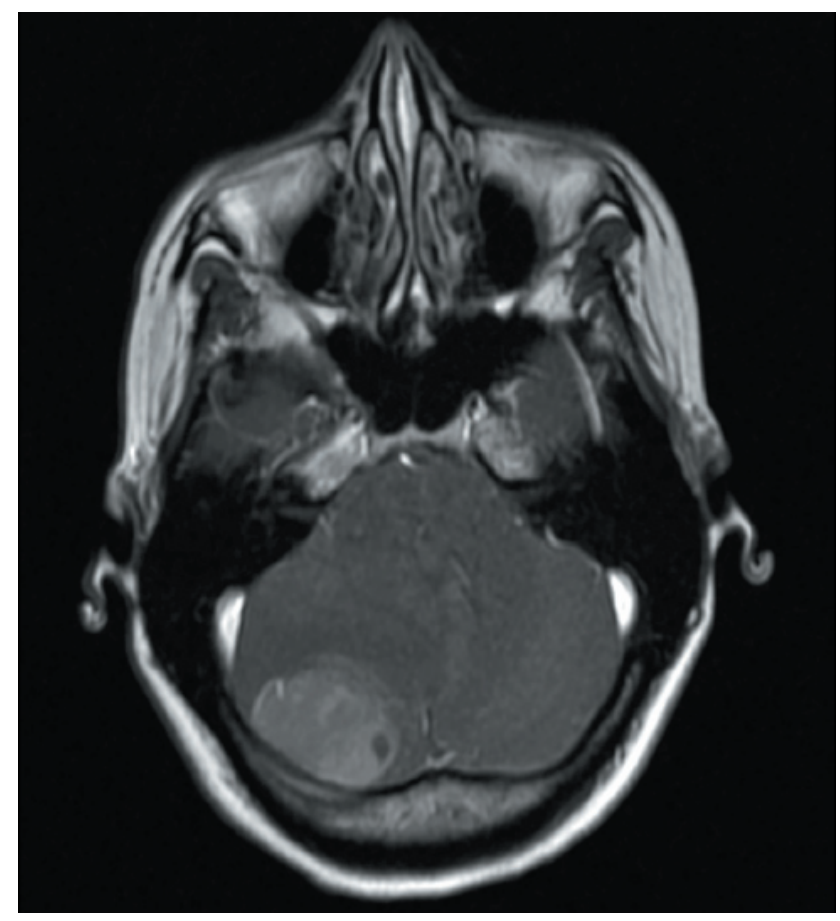

Figura 1. Corte axial de IRM cerebral, secuencia en T1 con contraste, donde identificamos una masa en hemisferio cerebeloso derecho, heterogénea, con irregularidad de sus bordes y con un tamaño de $29 \times 28 \times 36 \mathrm{~mm}$, con intensa captación de contraste sugestiva de meduloblastoma.
La técnica consistía en dos campos laterales y opuestos holocraneales y dos campos directos para el eje espinal con varios segmentos con fotones de 6/18 MV, seguido de una sobreimpresión en fosa posterior de dos campos laterales y opuestos conformados multiláminas con fotones de $6 \mathrm{MV}$, mediante un acelerador lineal.

Se analizó el seguimiento clínico de los pacientes, cuyo período comprendía desde la fecha del diagnóstico de la enfermedad hasta el final del estudio o el fallecimiento del paciente (si fuese el caso), tomando en cuenta el periodo de ingreso hospitalario y el seguimiento en consultas externas, resaltando las complicaciones postoperatorias observadas, y los resultados del control local y sistémico de la enfermedad con los tratamientos recibidos. Se consideró como recidiva tumoral la aparición de una nueva lesión (local y/o a distancia) después de finalizar el tratamiento correspondiente, destacando sus características y manejo, de acuerdo al número de episodios. Se empleó por último, el método Kaplan-Meier para estimar la supervivencia en los pacientes estudiados, utilizando el programa estadístico SPSS 15.0.

\section{Resultados}

La edad media de los pacientes fue 30.1 años, con un rango entre 18-56 años, distribuidos en 9 mujeres y 2 varones. Las características más importantes de los pacientes se agrupan en la tabla 1. Más del $80 \%$ de los pacientes

Tabla 1

Características de los pacientes con meduloblastomas

\begin{tabular}{|c|c|c|c|c|c|c|c|c|c|c|}
\hline \multicolumn{4}{|c|}{ Pacientes } & \multicolumn{7}{|c|}{ Características } \\
\hline & Sexo & Edad & $\begin{array}{l}\text { Año del } \\
\text { diagnós- } \\
\text { tico }\end{array}$ & $\mathrm{HIC}$ & $\begin{array}{l}\text { Síndro- } \\
\text { me cere- } \\
\text { beloso }\end{array}$ & $\begin{array}{l}\text { Duración } \\
\text { de los } \\
\text { síntomas }\end{array}$ & $\begin{array}{l}\text { Estado } \\
\text { preope- } \\
\text { ratorio }\end{array}$ & $\begin{array}{l}\text { Locali- } \\
\text { zación }\end{array}$ & Histología & $\begin{array}{l}\text { Estadiaje } \\
\text { según } \\
\text { Chang }\end{array}$ \\
\hline 1 & M & 19 & 2009 & si & si & $2-3$ & 4 & $\mathrm{HCI}$ & Desmoplásico & T3a-M0 \\
\hline 2 & $\mathrm{M}$ & 44 & 2009 & no & si & $<1$ & 2 & Línea media & Desmoplásico & T2-M0 \\
\hline 3 & $\mathrm{M}$ & 25 & 2008 & si & si & $2-3$ & 2 & $\mathrm{HCI}$ & Clásico & T3a-M0 \\
\hline 4 & M & 32 & 2008 & si & no & $<1$ & 2 & $\mathrm{HCI}$ & Clásico & T2-M1 \\
\hline 5 & $\mathrm{M}$ & 34 & 2008 & si & no & $1-2$ & 2 & Línea media & Desmoplásico & T3a-M0 \\
\hline 6 & $\mathrm{M}$ & 28 & 2005 & no & si & $<1$ & 2 & $\mathrm{HCI}$ & Clásico & T2-M0 \\
\hline 7 & $\mathrm{M}$ & 56 & 2004 & si & si & $<1$ & 2 & $\mathrm{HCI}$ & Clásico & T2-M0 \\
\hline 8 & $\mathrm{~V}$ & 34 & 2002 & si & no & $1-2$ & 2 & $\mathrm{HCI}$ & Desmoplásico & T2-M0 \\
\hline 9 & $\mathrm{M}$ & 18 & 1997 & si & si & $<1$ & 2 & Línea media & Clásico & T1-M0 \\
\hline 10 & $\mathrm{M}$ & 18 & 1988 & si & no & $2-3$ & 3 & Línea media & Clásico & T3-M0 \\
\hline 11 & $\mathrm{~V}$ & 24 & 1984 & si & no & $1-2$ & 3 & $\mathrm{HCI}$ & Desmoplásico & $\mathrm{T} 2-\mathrm{M} 0$ \\
\hline
\end{tabular}

HIC: hipertensión intracraneal. HCI: hemisferio cerebeloso izquierdo. HCD: hemisferio cerebeloso derecho. 
manifestaron clínica de hipertensión intracraneal, mientras el $54.5 \%$ presentaron síndrome cerebeloso, siendo la evolución de los síntomas menor a un mes al momento del diagnóstico en el $45.4 \%$ de los casos. El tumor estuvo localizado en algunos de los hemisferios cerebelosos en el $63.6 \%$ de los pacientes (figura 1). Tras el estudio histológico, las técnicas de inmunohistoquimia realizadas a 9 pacientes mostraron positividad para sinaptofisina y GFAP en 6 muestras y enolasa neuroespecífica en 5, con un índice de proliferación celular medido con Ki67 entre 30 y $90 \%$. De esta manera se diagnosticaron 6 pacientes con variedad clásica de meduloblastoma y 5 con variedad desmoplásica.

Fue necesario la colocación de una válvula de derivación ventriculoperitoneal (DVP) previa a la cirugía de exéresis tumoral en 3 pacientes por un cuadro de hidrocefalia obstructiva. La tabla 2 resume el tratamiento aplicado a los pacientes. El grado de resección quirúrgica fue total en 8 pacientes ( $72.7 \%$ ) y subtotal en $3(27.2 \%)$, confirmada en 6 casos mediante IRM y en el resto mediante TC cerebral realizados en las primeras 72 horas del postoperatorio. Se observaron complicaciones postoperatorias tempranas (durante la primera semana) en 3 casos: infección superficial de herida operatoria resuelta con antibioterapia intravenosa, fístula de LCR a trasvés de la herida que requirió reintervención quirúrgica para su cierre y un caso de hidrocefalia arreasortiva que requirió la colocación de válvula de DVP; por otro lado un paciente presentó un absceso cerebeloso como complicación tardía (al mes de la primera intervención) que requirió evacuación quirúrgica más antibioterapia intravenosa.

Todos los pacientes recibieron RT adyuvante, cuyo inicio se dilató entre 1 y 5 meses según la necesidad o no de QT adyuvante previa a la misma. Se administraron dosis de radiación al eje craneoespinal entre 30-36 Gy, y una sobreimpresión en fosa posterior entre 20-26 Gy. De esta manera, la dosis de radiación total administrada a nivel de la fosa posterior fue entre 54-56 Gy, con un ritmo de 1.8 Gy/sesión, 5 veces a la semana.

Posterior a la exéresis tumoral inicial, los 6 pacientes mas recientes de nuestra serie recibieron QT adyuvante con cisplatino (CDDP) y etopósido (VP-16), aplicando 3 ciclos de tratamiento previo a la RT. De estos pacientes, sólo 4 recibieron entre 2 y 3 nuevos ciclos una vez finalizada la misma. La toxicidad más frecuente relacionada a la radiación de estos enfermos fue la alteración hematológica, presente en todos los pacientes, con distintas maneras de presentación: neutropenia (con o sin leucopenia), plaquetopenia o anemia, resaltando 2 casos de aplasia medular transitoria. Estas complicaciones fueron responsables del retraso, ajuste de dosis o suspensión del tratamiento quimioterápico posterior en dichos pacientes. De esta manera, la totalidad de los ciclos de quimioterapia administrados al culminar el tratamiento adyuvante fue: 6 ciclos

Tabla 2

Tratamiento de pacientes con meduloblastoma

\begin{tabular}{|c|c|c|c|c|r|}
\hline \multirow{2}{*}{ Pacientes } & \multicolumn{5}{|c|}{ Tratamiento } \\
\hline & $\begin{array}{c}\text { Cirugía: grado } \\
\text { de resección }\end{array}$ & $\begin{array}{c}\text { RT Craneo- } \\
\text { espinal (Gy) }\end{array}$ & $\begin{array}{c}\text { Sobreimpresión } \\
\text { en fosa posterior (Gy) }\end{array}$ & Quimioterapia & $\begin{array}{c}\text { Año del } \\
\text { diagnóstico }\end{array}$ \\
\hline 1 & Total & 36 & 20 & CDDP+VP16 (Pre-RT) & 2009 \\
\hline 2 & Total & 29 & 27 & CDDP+VP16 (Pre y Post-RT) & 2009 \\
\hline 3 & Total & 30 & 25 & CDDP+VP16 (Pre-RT) & 2008 \\
\hline 4 & Total & 36 & 20 & CDDP+VP16 (Pre y Post-RT) & 2008 \\
\hline 5 & Subtotal & 34 & 20 & CDDP+VP16 (Pre y Post-RT) & 2008 \\
\hline 6 & Subtotal & 36 & 20 & CDDP+VP16 (Pre y Post-RT) & 2005 \\
\hline 7 & Total & 36 & 20 & no & 2004 \\
\hline 8 & Total & 36 & 20 & no & 2002 \\
\hline 9 & Total & 36 & 20 & no & 1997 \\
\hline 10 & Subtotal & 34 & 20 & no & 1988 \\
\hline 11 & Total & 34 & 20 & 1984 \\
\hline
\end{tabular}

RT: radioterapia. CDDP: cisplatino. VP-16: etopósido. 
en 2 pacientes, 5 en 2 pacientes y 3 en los otros 2 . En los 5 pacientes que no recibieron QT, la toxicidad hematológica postradiación tuvo un menor impacto, al observarse 2 casos de anemia y un paciente con aplasia transitoria.

En la tabla 3 se encuentran las características, patrones de recidiva del tumor y su manejo en los pacientes estudiados. De esta manera se observaron recidivas en el $36.3 \%$ de los pacientes $(n=4)$ durante el seguimiento. El tiempo medio de aparición del primer episodio de recidiva (y en uno de los casos, la única) en los pacientes fue 35.2 meses y la mediana fue 30 meses. De ellos, un paciente había recibido previamente tratamiento con cirugía con resección total, más RT y QT adyuvante, otros dos con cirugía con resección total más RT adyuvante, y un cuarto paciente en el cual la resección primaria había sido subtotal seguida de RT adyuvante. El sitio más frecuente de recidiva fue en la fosa posterior, específicamente en la misma localización del tumor original.

En 6 episodios de recidivas tumorales (in situ o en médula espinal) el tratamiento empleado fue la cirugía con exéresis lo mas amplia posible seguido en 5 de los mismos de QT adyuvante, empleando diferentes regímenes incluyendo genoxal/vincristina, CDDP/VP-16 y lomustina/ temozolomida. Cabe reseñar 2 pacientes que fallecen tras una tercera recidiva de la enfermedad luego de descartarse tratamientos agresivos por el pobre estado funcional $\mathrm{y}$, por otro lado, un paciente en el cual se decidió la opción de tratamiento con radiocirugía tras su primer episodio de recidiva, con lo cual se consiguió retrasar la progresión de su enfermedad durante 1 año. Se observaron recidivas con afectación de médula espinal en 2 pacientes: uno de los cuales fue sometido en dos ocasiones a tratamiento quirúrgico resectivo (total en la primera recidiva y subtotal en la segunda recidiva) más QT adyuvante, con lo cual se consiguió retrasar la progresión de la enfermedad y prolongar la supervivencia durante 18 meses aproximadamente y otro paciente que presentó afectación tumoral multicéntrica durante su tercer episodio de recidiva, a quien se le administró QT de rescate (CDDP + ciclofosfamida) con escaso éxito, ya que falleció pocas semanas después por progresión de su enfermedad.

De esta manera, tras un tiempo medio de seguimiento de los pacientes de 57.6 meses, con un rango entre 13-121 meses, 3 pacientes han fallecido por progresión de la enfermedad, encontrándose un tiempo medio de supervivencia estimada de 100.2 meses con un intervalo de confianza para la media al $95 \%$ entre 82.9 y 117.6 meses. En la figura 2 se muestra la curva de supervivencia de estos pacientes, en la cual se puede apreciar que todos los pacientes estudiados se encuentran vivos a los 4 años de seguimiento, con un índice de supervivencia estimado a los 5 y 8 años de seguimiento del 84 y $56 \%$ respectivamente.

Tabla 3

Patrones de recidiva de meduloblastomas y tratamiento

\begin{tabular}{|c|c|c|c|c|c|}
\hline Pacientes & $\begin{array}{l}\text { Edad } \\
\text { (años) }\end{array}$ & $\begin{array}{c}\text { Tratamiento } \\
\text { previo }\end{array}$ & $\begin{array}{l}\text { Tiempo y } \\
\text { número de } \\
\text { recidivas }\end{array}$ & Sitio de Recidiva & Tratamiento \\
\hline 1 & 32 & $\begin{array}{c}\text { Cirugía }+ \text { RT } \\
+\mathrm{QT}\end{array}$ & $\mathrm{R} 1: 20$ meses & In Situ & $\begin{array}{c}\text { Cirugía + QT (Genoxal + } \\
\text { Vincristina) }\end{array}$ \\
\hline 2 & 34 & Cirugía + RT & $\begin{array}{l}\text { R1: } 40 \text { meses } \\
\text { R2: } 12 \text { meses } \\
\text { R3: } 6 \text { meses }\end{array}$ & Médula espinal & $\begin{array}{l}\text { Cirugía + QT (CDDP + VP-16) } \\
\text { Cirugía + QT (Lomustina + } \\
\text { Temozolomida) } \\
\text { Cuidados paliativos * } \dagger\end{array}$ \\
\hline 3 & 24 & Cirugía + RT & $\begin{array}{l}\mathrm{R} 1: 9 \text { meses } \\
\mathrm{R} 2: 63 \text { meses } \\
\mathrm{R} 3: 24 \text { meses }\end{array}$ & In Situ & $\begin{array}{c}\text { Cirugía + RT } \\
\text { Cirugía + RT + QT (CDDP + VP-16) } \\
\text { Cuidados paliativos } * \dagger\end{array}$ \\
\hline 4 & 18 & Cirugía + RT & $\begin{array}{l}\mathrm{R} 1: 72 \text { meses } \\
\mathrm{R} 2: 12 \text { meses } \\
\mathrm{R} 3: 20 \text { meses }\end{array}$ & $\begin{array}{c}\text { In Situ } \\
\text { In Situ }+ \\
\text { Médula espinal }\end{array}$ & $\begin{array}{c}\text { Radiocirugía } \\
\text { Cirugía + QT (CDDP + VP-16) } \\
\text { QT (CDDP + ciclofosfamida }) \dagger\end{array}$ \\
\hline
\end{tabular}

* Tratamiento sintomático debido al mal estado general del paciente.

$\dagger$ Fallece por progresión de la enfermedad.

R1: primera recidiva. R2: segunda recidiva. R3: tercera recidiva.

RT: radioterapia. QT: quimioterapia. CDDP: cisplatino. VP-16: etopósido. 


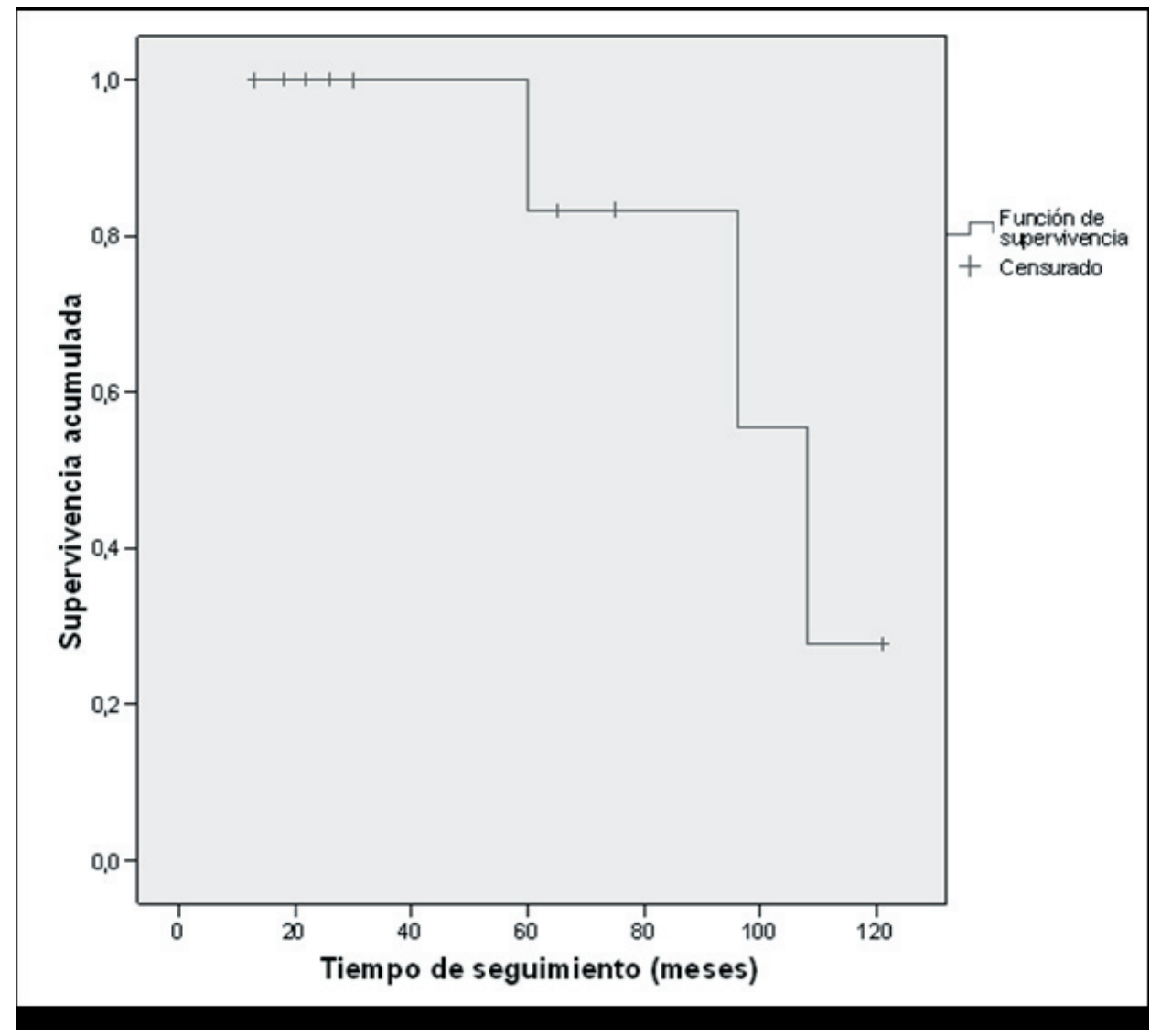

Figura 2. Curva de Supervivencia de los pacientes con meduloblastomas.

Al final del estudio, los pacientes vivos se encuentran con buen control de la enfermedad (sin cambios significativos en pruebas de imagen del neuroeje a lo largo del tiempo) y continúan en régimen de visitas en consultas externas de las distintas especialidades implicadas. De ellos, 6 se encuentran asintomáticos y hacen vida normal (estado funcional final según la escala de la ECOG de 1) y en dos casos son totalmente ambulatorios y realizan actividades de la vida diaria, aunque se encuentran con algunos síntomas residuales, en especial ataxia leve (estado funcional final de 2).

\section{Discusión}

Los meduloblastomas del adulto constituyen una entidad maligna infrecuente ${ }^{5,10}$. La incidencia europea anual (estandarizada a nivel mundial) es alrededor de 1.1 por millón en varones y 0.8 por millón en mujeres dentro de la población adulta ${ }^{39}$. Por otro lado, al tratarse de tumores cerebrales muy comunes en la población pediátrica, cerca del $70 \%$ de los casos se diagnostican antes de los 15 años de edad ${ }^{5}$, con un pico de incidencia más importante entre los 3-6 años. Esto es seguido de un segundo pico menos significativo (25\%) entre los $15-44$ años ${ }^{40}$, en concordancia con los pacientes de este estudio, de los cuales el $90 \%$ pertenecen a este grupo, con una edad media de 30.1 años. A pesar de encontrar mayor incidencia en hombres en la literatura ${ }^{20,39}$, el $81.8 \%$ de los pacientes fueron mujeres, sin encontrar una justificación clara de este hallazgo.

La mayoría de los tumores se manifestaron como un síndrome de hipertensión intracraneal, similar a lo referido por otros autores y coincidiendo con la población pediátrica $^{5,13,43}$. Entre otros hallazgos en acuerdo con la literatura se encuentran que más de la mitad de los pacientes tuvieron tumores localizados en uno de los hemisferios cerebelosos ${ }^{3,4,9,11}$, con una alta proporción de tumores con variedad histopatológica desmoplásica (figura 3$)^{8,11,23,33}$.

En los 3 pacientes más antiguos de la serie (entre 19841997), se decidió colocar una DVP para el tratamiento de la hidrocefalia obstructiva previo a la resección quirúrgica de la lesión, como parte de los protocolos de actuación para el momento. Sin embargo, en los últimos años se observa la tendencia de manejar inicialmente este tipo de pacientes, con buen nivel de consciencia, con medidas conservadoras (corticoides) seguido de una intervención quirúrgica resectiva al más corto plazo, reservando las técnicas de derivación de LCR para aquellos pacientes con deterioro 


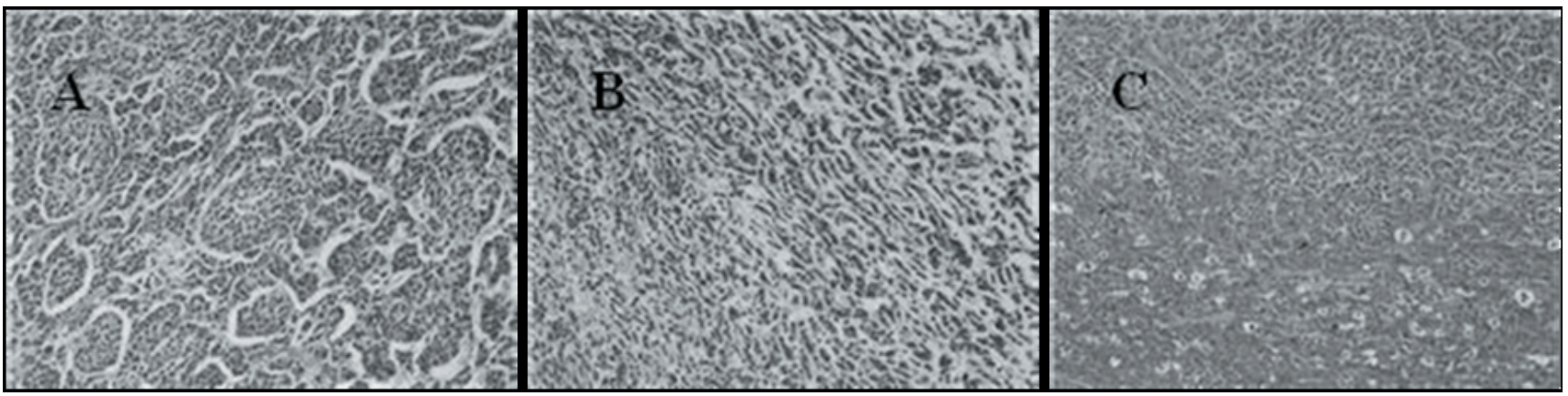

Figura 3. Características histológicas del meduloblastoma desmoplásico (Paciente 1 de la muestra). A. Se observan zonas nodulares pálidas alternas con zonas celulares (hematoxilina y eosina). B. Area desmoplásica, con disposición en fila de la celularidad y discreto amoldamiento nuclear (hematoxilina y eosina). C. Tinción inmunohistoquímica que muestra positividad frente a sinaptofisina, característica de este tumor.

neurológico progresivo por hipertensión intracraneal, en cuyo caso se plantea como primera opción si fuese posible, la ventriculostomía endoscópica o en su defecto un drenaje ventricular externo transitorio (lo cual no fue necesario en el resto de los pacientes de nuestra serie), disminuyendo posibles complicaciones propias de enfermos portadores de DVP, en especial problemas de malfunción por obstrucción y el riesgo teórico de diseminación peritoneal, la cual, aunque no se observó en ninguno de los casos, ha sido descrita por algunos autores ${ }^{6}$.

La cirugía constituye un rol crucial en el tratamiento ${ }^{27,28}$, encontrándose en la literatura numerosos estudios que resaltan la importancia pronóstica de la resección tumoral

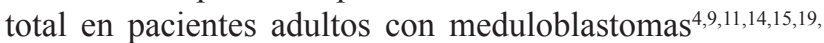
$33,24,28,29,34,42$, hallazgo igualmente reportado en poblaciones pediátricas $^{2,12,30,38,49}$. De esta manera, en nuestra serie se consiguió la exéresis tumoral mayor del $90 \%$ en 8 pacientes $(72.72 \%)$, favorecido en la mayoría de los casos por la localización hemisférica de la lesión, y que fue confirmada por imágenes de TC cerebral en 6 pacientes y de IRM cerebral en los otros 2, realizadas durante el postoperatorio temprano. Cabe destacar que al finalizar el estudio, 6 de ellos se encuentran vivos y con enfermedad controlada. Las complicaciones observadas en los pacientes intervenidos incluyeron la infección, la fístula de LCR y la hidrocefalia, propias de este tipo de patología y abordaje quirúrgico de la fosa craneal posterior ${ }^{22,47}$.

La RT ha mejorado considerablemente el pronóstico de pacientes con meduloblastomas en los últimos 40 años, empleándose de manera estándar después de la cirugía, con disminución de su recidiva y aumento de la supervivencia tanto en niños como en adultos, utilizando actualmente sistemas de planificación del tratamiento conformado con el objetivo de respetar, en la manera de lo posible, el parénquima normal ${ }^{5,41}$. Motivado por la elevada tendencia de diseminación a través del LCR de estos tumores y de acuerdo a lo descrito por otros autores ${ }^{11,23,31,43}$, en nuestro centro se han aplicado protocolos con dosis de radiación al eje craneoespinal entre 30-36 Gy, y una sobreimpresión en fosa posterior entre 20-26 Gy; por lo tanto se administraron dosis totales a nivel de la fosa posterior entre 54-56 Gy, ya que se ha demostrado que dosis menores de radiación se relacionan con un peor pronóstico tanto en adultos ${ }^{1,23,25}$ como en niños ${ }^{48}$.

En niños con meduloblastoma la QT adyuvante ha demostrado con un nivel de evidencia grado 1 un aumento de la supervivencia cuando se administra en conjunto con la cirugía y la $\mathrm{RT}^{46}$. En adultos, su papel no está totalmente definido, observando, por ejemplo en algunos estudios retrospectivos, índices de supervivencia a los 5 años que varían entre el 26 y $83 \%$ independientes de su uso como adyuvancia oncológica, ${ }^{4,8,15,19,41}$. Aún así, es una realidad que, por extrapolación de la población pediátrica, cada vez se incrementa su uso en las series de pacientes adultos publicadas en los últimos años ${ }^{8,11,25}$, recomendándose en especial en aquellos casos con resecciones tumorales incompletas, estadios de Chan > T3a y diseminación a través del LCR ${ }^{5}$. En base a esto, y luego de ser evaluados en comités neurooncológicos correspondientes, 6 pacientes del presente estudio fueron sometidos a tratamiento quimioterápico posterior a la resección tumoral primaria.

Existe una heterogeneidad de protocolos en la literatura, sin embargo se suele incluir CDDP, VP-16 y ciclofosfamida, siendo los dos primeros los empleados como primera línea en los pacientes de la presente serie. Por otro lado, el protocolo aplicado en nuestro centro incluye el inicio de los ciclos de QT previos a la radiación, con el objetivo de obtener mayor control con el tratamiento con RT, conociendo que el retraso en su inicio no supone la progresión de la enfermedad ${ }^{16}$, aunque con la desventaja de una mayor toxicidad hematológica observada durante la radiación, ya descrito en estudios ${ }^{26}$, con el riesgo que esto conlleva a que se retrasen o condicionen en algunos casos la culminación de los ciclos de QT postradiación, como se ha evidenciado 
en ciertos pacientes de nuestra serie.

Durante un tiempo de seguimiento entre 13-121 meses, con una media de 57.6 meses, 4 de 11 pacientes presentaron recidivas de la enfermedad, que ocurrieron en la mitad de los casos posterior a los 2 años del tratamiento inicial, hallazgos que se diferencian de la población pediátrica, en la cual están descritas un $75 \%$ de recidivas en los primeros 2 años ${ }^{17}$. En este sentido cabe destacar que la literatura reporta como hallazgo común índices tardíos de recidivas en la población adulta ${ }^{4,5,8,11,20,24,31,32}$, por lo tanto la vigilancia de estos pacientes a largo plazo es importante. En el presente estudio el lecho quirúrgico original constituyó el sitio más frecuente de recidiva, en concordancia con otros autores ${ }^{4,8,9,11,23,31}$ y similar a lo descrito en la población pediátrica.

No existen datos prospectivos disponibles para establecer el mejor tratamiento en los casos de recidiva tumoral ${ }^{5}$, sin embargo se debe considerar preferiblemente la opción quirúrgica, en especial en casos con recidivas locales, como lo observado en esta serie. Está descrito el uso de distintos quimioterápicos, incluyendo genoxal, viscristina, lomustina o temozolomida ${ }^{7,11,42}$, como se ha reflejado en los pacientes del presente estudio. También recientemente se ha incorporado la opción de la radiocirugía al tratamiento adyuvante, la cual fue aplicada a una paciente de la actual muestra, con resultados esperanzadores 18 , consiguiendo en este caso retrasar la progresión de la enfermedad durante un año.

La bibliografía clasifica los pacientes con meduloblastomas en dos grupos: aquellos considerados de bajo riesgo si pertenecen a estadios de Chang T1, T2, T3a, M0, sin enfermedad residual después de la cirugía, y los considerados de alto riesgo que incluyen a aquellos con estadios $\geq \mathrm{T} 3 \mathrm{~b}, \geq \mathrm{M} 1$ o con restos postquirúrgicos. En general, el tiempo de supervivencia a los 5 años de un paciente adulto con meduloblastoma es del $25 \%$ en caso de ser de alto riesgo, y del 75\% si se trata de un paciente de bajo riesgo ${ }^{16}$. En este sentido, destacan los hallazgos de Sunyach ${ }^{45}$ sobre una serie de 253 pacientes donde informan una supervivencia a los 10 años del $68 \%$ en pacientes de bajo riesgo y un $46 \%$ en los de alto riesgo. Greenberg ${ }^{21}$ encontró una supervivencia libre de progresión de la enfermedad a los 5 años del $45 \%$, en comparación del $80 \%$ referida por Malheiros ${ }^{36}$, $83 \%$ referida por $\mathrm{Chan}^{11}$ y $65 \%$ por Spreafico ${ }^{44}$. En Alemania, se analizaron 56 pacientes quienes recibieron quimioterapia adicional según el protocolo HIT'91 y se encontró una supervivencia libre de enfermedad a los 5 años del $67 \%$, con peor pronóstico en estadio M3 (54\%) que en estadio M0 $(71 \%)^{5}$. A pesar de contar con pocos pacientes (lo cual no favorece el estudio por subgrupos de riesgo), en la presente serie, similar a lo descrito por otros autores ${ }^{11,36}$ se observó un índice de supervivencia general estimado a los 5 años del 84\% empleando tratamiento quirúrgico resectivo más tratamiento oncológico adyuvante (RT con o sin QT); contando con 8 pacientes vivos al final del estudio con buen control de la enfermedad.

\section{Conclusiones}

En base a nuestros resultados, se observa que los meduloblastomas del adulto son tumores poco frecuentes, con predominio de localización hemisférica y aumento de variedad histológica desmoplásica al compararlo con la población pediátrica. El alto índice de supervivencia presente en los pacientes de esta serie apoya la importancia de conseguir una resección quirúrgica total, seguida de tratamiento oncológico adyuvante tan pronto sea posible, destacando el rol ya establecido que posee la RT sobre el eje craneoespinal. Llama la atención, en este sentido, el aumento de los casos que han recibido QT adyuvante postquirúrgica en los últimos años, observando un buen control de la enfermedad en estos pacientes a corto y mediano plazo, a pesar de la evidencia de toxicidad, principalmente hematológica, al combinarse luego con la RT. Sin embargo, son necesarios estudios con mayor número de pacientes y a largo plazo (tomando en cuenta la presencia de recidivas más tardías que en niños) para encontrar mejor evidencia sobre la importancia de la quimioterapia en estos enfermos, incluyendo el momento de su administración (pre y/o post radioterapia) y el número de ciclos. El objetivo final es proporcionar el mejor manejo integral y óptimo (cirugía, RT y QT) dentro de un equipo multidisciplinar para retrasar la progresión/recidiva de esta maligna enfermedad y mejorar la supervivencia y calidad de vida de estos pacientes.

\section{Bibliografía}

1. Abacioglu, U., Uzel, O., Sengoz, M., Turkan, S., Ober, A.: Medulloblastoma in adults: treatment results and prognostic factors. Int J Radiat Oncol Biol Phys 2002; 54: 855-860.

2. Albright, A.L., Wisoff, J.H., Zeltzer, P.M., Boyett, J.M., Rorke, L.B., Stanley, P.: Effects of medulloblastoma resections on outcome in children: A report from the Children's Cancer Group. Neurosurgery 1996; 38: 265-271.

3. Aragones, M.P., Magallon, R., Piqueras, C., Ley, L., Vaquero, J., Bravo, G.: Medulloblastoma in adulthood: Prognostic factors influencing survival and recurrence. Acta Neurochir (Wien) 1994; 127: 65-68.

4. Bloom, H.J., Bessell, E.M.: Medulloblastoma in adults: A review of 47 patients treated between 1952 and 1981. Int J Radiat Oncol Biol Phys 1990; 18: 763-772.

5. Brandes, A., Franceschi, E., Tosoni, A., Reni, M., Gatta, G., Vecht, Ch., et al.: Adult neuroectodermal tumors of posterior fossa (medulloblastoma) and of supratentorial sites (stPNET). Critical Reviews in Oncology/Hematology 2009; 
71: $165-179$.

6. Brandes, A., Palmesano, V., Monfardini, S.: Medulloblastoma in adults: clinical characteristics and treatment. Canc Treat Rev 1999; 25: 3-12.

7. Cakar, M., Aksoy, S., Kilickap, S., Harputluoglu, H., Erman, M.: Procarbazine, lomustine, vincristine combination may be effective in adult medulloblastoma patients with systemic metastases. J Neurooncol 2005; 75: 233-234.

8. Carrie, C., Lasset, C., Alapetite, C., Haie-Meder, C., Hoffstetter, S., Demaille, M.C., et al.: Multivariate analysis of prognostic factors in adult patients with medulloblastoma: Retrospective study of 156 patients. Cancer 1994; 74: 23522360.

9. Carrie, C., Lasset, C., Blay, JY., Negrier, S., Bouffet, E., Barbet, N., et al.: Medulloblastoma in adults: Survival and prognostic factors. Radiother Oncol 1993; 29: 301-307.

10. CBTRUS Executive Team. Central Brain Tumor Registry of the United States - Statistical report 1992-1997, years data collected. Chicago; CBTRUS, 2000.

11. Chan, A.W., Tarbell, N.J., Black, P.M., Louis, D.N., Frosch, M.P., Ancukiewicz, M., et al.: Adult medulloblastoma: prognostic factors and patterns of relapse. Neurosurgery 2000; 47: 623-632.

12. Del Charco, J.O., Bolek, T.W., McCollough, W.M., Maria, B.L., Kedar, A., Braylan, R.C., et al.: Medulloblastoma: Time-dose relationship based on a 30 -year review. Int $\mathrm{J}$ Radiat Oncol Biol Phys 1998; 42: 147-154.

13. Eisenstat, D.D.: Clinical management of medulloblastoma in adults. Expert Rev Anticancer Ther 2004; 4: 795-802.

14. Ferrante, L., Mastronardi, L., Celli, P., Acqui, M., Cervoni, L., Fortuna, A.: Medulloblastoma in adulthood. J Neurosurg Sci 1991; 35: 23-30.

15. Frost, P.J., Laperriere, N.J., Wong, C.S., Milosevic, M.F., Simpson, W.J.S., Pintilie, M.: Medulloblastoma in adults. Int J Radiat Oncol Biol Phys 1995; 32: 951-957.

16. Gandía González, M.L., Isla Guerrero, A., Pérez Zamarrón, A., Gómez Sierra, A.: Meduloblastomas del adulto. En Grupo de neurooncología de la sociedad española de neurocirugía (eds). Tumores de fosa posterior. Barcelona; Permanyer, 2008; pp. 83-94.

17. Gelabert González, M., Serramito García, R., Reyes Santías, R.M.: Meduloblastomas infantiles. En Grupo de neurooncología de la sociedad española de neurocirugía (eds). Tumores de fosa posterior. Barcelona; Permanyer, 2008; pp. 75-82.

18. Germanwala, A.V., Mai, J.C., Tomycz, N.D., Niranjan, A., Flickinger, J.C., Kondziolka, D., et al.: Boost gamma knife surgery during multimodality management of adult medulloblastoma. J Neurosurgery 2008; 108: 204-209.

19. Giordana, M.T., Cavalla, P., Chio, A., Marino, S., Soffietti, R., Vigliani, M.C., et al.: Prognostic factors in adult medulloblastoma: a clinico-pathologic study. Tumori 1995; 81: 338-346.
20. Giordana, M.T., Schiffer, P., Lanotte, M., Girardi, P., Chiò, A.: Epidemiology of adult medulloblastoma. Int J Cancer 1999; 80: 689-692.

21. Greenberg, H.S., Chamberlain, M.C., Glantz, M.J., Wang, S.: Adult medulloblastoma: multiagent chemotherapy. Neuro Oncol 2001; 3: 29-34.

22. Greenberg, M.S.: Handbook of neurosurgery. Florida; Thieme, 2006; pp. 604-607.

23. Haie, C., Schlienger, M., Constans, J.P., Meder, J.F., Reynaud, A., Ghenim, C.: Results of radiation treatment of medulloblastoma in adults. Int J Radiat Oncol Biol Phys 1985; 11: 2051-2056.

24. Hartsell, W.F., Montag, A.G., Lydon, J., Galinsky, D.L., Sarin, P.: Treatment of medulloblastoma in adults. Am J Clin Oncol 1992; 15: 207-211.

25. Herrlinger, U., Steinbrecher, A., Rieger, J., Hau, P., Kortmann, R.D., Meyermann, R., et al.: Adult medulloblastoma: prognostic factors and response to therapy at diagnosis and at relapse. J Neurol 2005; 252: 291-299.

26. Hesham, H.A.: Pre-Irradiation Chemotherapy in High Risk Medulloblastoma. J Egypt Natl Canc Inst 2006; 18: $357-$ 362 .

27. Hughes, P.G.: Cerebellar medulloblastoma in adults. J Neurosurg 1984; 60: 994-997.

28. Iaconetta, G., Lamaida, E., Rossi, A.: Cerebellar medulloblastoma in adults: report of 15 cases and review of the literature. Acta Neurol (Napoli) 1994; 16: 38-45.

29. Ildan, F., Cetinalp, E., Bagdatoglu, H.: Cerebellar medulloblastoma in adults. Neurosurg Rev 1994; 17: 205209.

30. Jenkin, D., Goddard, K., Armstrong, D., Becker, L., Berry, M., Chan, H., et al.: Posterior fossa medulloblastoma in childhood: Treatment results and a proposal for a new staging system. Int J Radiat Oncol Biol Phys 1990; 19: 265-274.

31. Kopelson, G, Linggood, R.M., Kleinman, G.M.: Medulloblastoma in adults: improved survival with supervoltage radiation therapy. Cancer 1982; 49: 1334-1337.

32. Krampulz, T., Hans, V.H., Oppel, F., Dietrich, U., Puchner, M.J.: Long-term relapse-free survival with supratentorial primitive neuroectodermal tumour in an adult: a case report. J Neurooncol 2006; 77: 291-294.

33. Kunschner, L.J., Kuttesch, J., Hess, K., Yung, W.K.A.: Survival and recurrence factors in adult medulloblastoma: the M.D. Anderson Cancer Center experience from 1978 to 1998. Neuro Oncol 2001; 3: 167-173.

34. Le, Q.T., Weil, M.D., Wara, W.M., Lamborn, K., Prados, M.D., Edwards, M.S.B., et al.: Adult medulloblastoma: an analysis of survival and prognostic factors. Cancer $\mathrm{J}$ Sci Am 1997; 3: 238-245.

35. Louis, D.N., Ohgaki, H., Wiestler, O.D., Cavenee, W.K.: WHO Classification of Tumours of the Central Nervous System. Lyon; International Agency for Research on Cancer, 2007: pp. 132-140. 
36. Malheiro, S.M.F., Franco, C.M.R., Stavale, J.N., Santos, A.J., Borges, L.R.R., Pelaez, M.P., et al.: Medulloblastoma in adults: a series from Brazil. J Neurooncol 2002; 60: 247-253.

37. Menon, G., Krishnakumar, K., Nair, S.: Adult medulloblastoma: clinical profile and treatment results of 18 patients. $\mathrm{J}$ Clin Neuroscience 2008; 15: 122-126.

38. Merchant, T.E., Wang, M.H., Haida, T., Lindsley, K.L., Finlay, J., Dunkel, I.J., et al.: Medulloblastoma: Long-term results for patients treated with definitive radiation therapy during the computed tomography era. Int J Radiat Oncol Biol Phys 1996; 36: 29-35.

39. Parkin, D.M., Whelan, S.L., Ferlay, J., Teppo, L., Thomas, D.B.: Cancer incidence in five continents, vol. VIII. Lyon; International Agency for Research on Cancer, 2002.

40. Peris-Bonet, R., Martinez-Garcia, C., Lacour, B., Petrovich, S., Giner-Ripoll, B., Navajas, A., et al.: Childhood central nervous system tumours-incidence and survival in Europe (1978-1997): report from Automated Childhood Cancer Information System project. Eur J Cancer 2006; 42: 2064-2080.

41. Prados, M.D., Warnick, R.E., Wara, W.M., Larson, D.A., Lamborn, K., Wilson, C.B.: Medulloblastoma in adults. Int J Radiat Oncol Biol Phys 1995; 32: 1145-1152.

42. Rose, L.: Survival of patients with adult medulloblastoma: a population-based study. Cancer 2008; 112: 15681574.

43. Smee, R.I., Williams, J.R.: Medulloblastomas - Primitive neuroectodermal tumours in the adult population. J Med Imaging Radiat Oncol 2008; 52: 72-76.

44. Spreafico, F., Massimino, M., Gandola, L., Cefalo, G., Mazza, E., Landonio, G., et al.: Survival of adults treated for medulloblastoma using paediatric protocols. Eur J Cancer 2005; 41: 1304-1310.

45. Sunyach, M., Padovani, L., Murraciole, X., Alapetite,
C., Haie-Meder, C., Hoffstetter, S., et al.: Multivariate analysis of prognostic factors in adult medulloblastoma: retrospective multicen-tric study. Int J Radiat Oncol Biol Phys 2005; 63 (Suppl.): 67-68.

46. Taylor, R.E., Bailey, C.C., Robinson, K., Weston, C.L., Ellison, D., Ironside, J., et al.: Results of a randomized study of preradiation chemotherapy versus radiotherapy alone for nonmetastatic medulloblastoma: The International Society of Paediatric Oncology/United Kingdom Children's Cancer Study Group PNET-3 Study. J Clin Oncol 2003; 21: 15811591.

47. Tejada Solís, S., Díez Valle, R.: Complicaciones en la cirugía de la fosa posterior. En Grupo de neurooncología de la sociedad española de neurocirugía (Eds). Tumores de fosa posterior. Barcelona; Permanyer, 2008; pp. 59-64.

48. Thomas, P.R., Deutsch, M., Kepner, J.L., Boyett, J.M., Krischer, J., Aronin, P., et al.: Low-stage medulloblastoma: final analysis of trial comparing standard-dose with reduceddose neuraxis irradiation. J Clin Oncol 2000; 18: 3004-3011.

49. Zeltzer, P.M., Boyett, J.M., Finlay, J.L., Albright, A.L., Rorke, L.B., Milstein, J.M., et al.: Metastasis stage, adjuvant treatment, and residual tumor are prognostic factors for medulloblastoma in children: Conclusions from the Children's Cancer Group 921 randomized Phase III study. J Clin Oncol 1999; 17: 832-845.

Rodríguez-Mena, R.; Barbella-Aponte, R.A.; GallegoSánchez, J.M.; Barcia-Mariño, C.: Meduloblastomas del adulto: serie quirúrgica de 11 casos. Neurocirugía 2011; 22: 488-497.

Correspondencia: Dr. Ruben Rodríguez Mena. Servicio de Neurocirugía. Consorcio Hospital General Universitario de Valencia. Avda. Tres Cruces, 2. E-46014 Valencia.

E-mail: ruben.rod@gmail.com 